“(C) 2018 IEEE. Personal use of this material is permitted. Permission from IEEE must be obtained for all other uses, in any current or future media, including reprinting/republishing this material for advertising or promotional purposes, creating new collective works, for resale or redistribution to servers or lists, or reuse of any copyrighted component of this work in other works." 


\title{
Shaped Power Pattern Synthesis of a Linear Dipole Array by Element Rotation and Phase Optimization Using Dynamic Differential Evolution
}

\author{
Ming Li, Yanhui Liu, Member, IEEE, Y. Jay Guo, Fellow, IEEE
}

\begin{abstract}
A novel strategy for synthesizing shaped power pattern by using element rotation and phase optimization for a linear dipole array is presented. Element rotation can be considered as an array amplitude weighting technique, and it provides additional degrees of freedom in the pattern synthesis but potentially increases the cross-polarization level (XPL). In order to obtain a desired co-polarized shaped pattern with both the sidelobe level (SLL) and XPL constrained, we simultaneously optimize all the rotation angles and excitation phases of all the dipole elements by applying the dynamic differential evolution (DDE) algorithm. Two experiments for synthesizing flat-top and cosecant-squared patterns are conducted to validate the effectiveness and advantages of the proposed strategy.
\end{abstract}

Index Terms-Shaped power pattern, dipole rotation, crosspolarization level (XPL), dynamic differential evolution (DDE).

\section{INTRODUCTION}

A TENNA arrays with shaped power patterns have been widely applied in high-performance wireless communication and sensing systems. Many advanced shaped pattern synthesis methods have been presented in the past, such as the analytical techniques [1], [2], the iterative sampling method [3], alternating projection method [4], mathematical programming-based optimization techniques [5]-[8], and stochastic optimization algorithms [9]-[13]. Owing to the complexity of the shaped pattern synthesis, however, most of synthesis methods need to optimize both the excitation amplitudes and phases to achieve the desired pattern shape and sidelobe level. Despite their success in pattern shape control, they tend to result in a relatively complicated feeding network design especially when multiple unequal power dividers are required. Hence, a few methods such as those in [14]-[16] have tried to only optimize the excitation phases to achieve desired pattern shaping. Owing to the limited degrees of freedom, however, both the mainlobe shape accuracy and the sidelobe performance are considerably worse comparing the case when full control of both the excitation amplitudes and phases are employed. In [17], an effective deterministic method is

Manuscript received Xxx. This work was supported in part by Shenzhen Science and Technology Innovation Project under Grant No YCYJ 20170307141315473 and in part by Science and Technology Research Project of Fujian Province under Grant No 2017 I0017 (Corresponding author: Yanhui Liu).

M. Li and Y. Liu are with Institute of Electromagnetics and Acoustics, Xiamen University, Fujian 361005, China, and also with the Shenzhen Research Institute of Xiamen University, Shenzhen 518057, China (email: yanhuiliu@xmu.edu.cn).

Y. Jay Guo is with the Global Big Data Technologies Centre, University of Technology Sydney (UTS), NSW 2007, Australia. presented which combines element positions and phases to improve the shaped pattern approximation accuracy.

It is understood that using element rotation can change the power distributions of both the co- and cross-polarized components on a fixed observation plane. Hence, the element rotation technique can be considered as an alternative way of realizing amplitude weighting for antenna arrays. In [18] and [19], the element rotation technique was used to effectively reduce sidelobe levels of focused beam patterns for dipole antenna arrays. However, to be the best of our knowledge, such a technique has never been applied to improve the performance of shaped pattern synthesis in literature. In this paper, we will show that a desired co-polarized shaped power pattern with constrained sidelobe level (SLL) and cross-polarization level (XPL) can be obtained by finding appropriate element rotation angles and excitation phases for the case of dipole antenna arrays. Compared to the conventional shaped pattern array by using both the amplitude and phase weighting, the currently obtained array avoids usage of multiple unequal power dividers and significantly simplifies the feeding network.

Synthesizing rotation angles and excitation phases is a highly nonlinear problem. Stochastic optimization algorithms would be good choices in dealing with this problem because of their powerful search schemes to find the global optimum. Among them, the dynamic differential evolution (DDE) method is one of demonstrated powerful global optimizers. In this work, the DDE is chosen to simultaneously optimize the element rotation angles and excitation phases for obtaining a desired co-polarized shaped pattern with constrained XPL. In the DDE, if the generated trial individual is better than the corresponding target individual, it will replace the target individual and be immediately used in the following evolution instead of in the next generation in the conventional DE (CDE) [20]. This leads to a larger virtual population and quicker response to the change of population status. The reliability and efficiency of the DDE have already been verified in different antenna array synthesis applications [20][22]. Numerical results are given for synthesizing flat-top and cosecant-squared patterns by only element rotation and phase optimization of linear dipole arrays, and the results demonstrate the effectiveness and advantages of the proposed method.

It should be noted that the proposed method is involved in synthesizing vectorial pattern with cross-polarization suppression. Some early relevant studies are available in the literature, such as [23] for the cross-polarization and sidelobe 


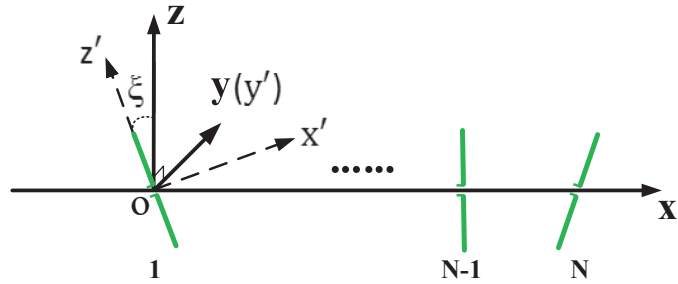

Fig. 1. The rotated dipole in a $N$-element array.

suppression by using appropriate subarray configuration with part of mirrored elements in dual linear polarization antenna arrays, and [24] where efficient co- and cross-polarization pattern synthesis is achieved by introducing two auxiliary phase patterns for reducing the vectorial power synthesis problem to a field synthesis one.

\section{Formulation AND Algorithm}

\section{A. Vectorial pattern of a dipole-rotated array}

For the shaped pattern synthesis of a dipole-rotated array, formulation of its co- and cross-polarized patterns is necessary. At first, consider a dipole with a rotation angle $\xi \in[-\pi, \pi]$ from $z$-axis in the global coordinate system (GCS) $x y z$, as shown in Fig.1. To facilitate the pattern analysis, a local coordinate system (LCS) $x^{\prime} y^{\prime} z^{\prime}$ is built such that the $z^{\prime}$-axis coincides with the dipole's orientation. Hence, the electrical field pattern of this dipole in the LCS can be written as

$$
\boldsymbol{a}\left(\theta^{\prime}, \phi^{\prime}\right)=\frac{\cos \left(\frac{\pi}{2} \cos \theta^{\prime}\right)}{\sin \theta^{\prime}} \hat{\boldsymbol{\theta}}^{\prime}
$$

where $\theta^{\prime}$ and $\phi^{\prime}$ are the angles measured from $z^{\prime}$-axis and $x^{\prime}$-axis, respectively, and $\hat{\boldsymbol{\theta}}^{\prime}$ is a unit vector in $\theta^{\prime}$-direction. In order to obtain the array pattern by summing all the field patterns of rotated dipoles in the same coordinate, we now transform the element pattern expression in the LCS to the one in the GCS via coordinate transformation. Note that in the LCS, the $\phi^{\prime}$-polarized component of the electrical field pattern does not exist, but in the GCS, both $\theta$ - and $\phi$-polarized components exist. They can be written by

$$
\begin{aligned}
a_{\theta}\left(\theta^{\prime}, \phi^{\prime}\right) & =\frac{\cos \left(\frac{\pi}{2} \cos \theta^{\prime}\right)}{\sin \theta^{\prime}} \hat{\boldsymbol{\theta}}^{\prime} \cdot \hat{\boldsymbol{\theta}} \\
a_{\phi}\left(\theta^{\prime}, \phi^{\prime}\right) & =\frac{\cos \left(\frac{\pi}{2} \cos \theta^{\prime}\right)}{\sin \theta^{\prime}} \hat{\boldsymbol{\theta}}^{\prime} \cdot \hat{\boldsymbol{\phi}} .
\end{aligned}
$$

In the above, $\hat{\boldsymbol{\theta}}^{\prime}$ in the LCS is given by

$$
\hat{\boldsymbol{\theta}}^{\prime}=\cos \theta^{\prime} \cos \phi^{\prime} \hat{\boldsymbol{x}}^{\prime}+\cos \theta^{\prime} \sin \phi^{\prime} \hat{\boldsymbol{y}}^{\prime}-\sin \theta^{\prime} \hat{\boldsymbol{z}}^{\prime} \text {. }
$$

By using coordinate transformation, it can be rewritten in the GCS as

$$
\begin{gathered}
\hat{\boldsymbol{\theta}}^{\prime}=\left(\cos \xi \cos \theta^{\prime} \cos \phi^{\prime}+\sin \xi \sin \theta^{\prime}\right) \hat{\boldsymbol{x}}+\cos \theta^{\prime} \sin \phi^{\prime} \hat{\boldsymbol{y}} \\
+\left(\sin \xi \cos \theta^{\prime} \cos \phi^{\prime}-\cos \xi \sin \theta^{\prime}\right) \hat{\boldsymbol{z}} .
\end{gathered}
$$

Hence, by considering the component expressions of $\hat{\boldsymbol{\theta}}$ and $\hat{\boldsymbol{\phi}}$, we can obtain that

$$
\begin{aligned}
a_{\theta}\left(\theta^{\prime}, \phi^{\prime}\right)= & \frac{\cos \left(\frac{\pi}{2} \cos \theta^{\prime}\right)}{\sin \theta^{\prime}}\left[\operatorname { c o s } \theta \operatorname { c o s } \phi \left(\cos \xi \cos \theta^{\prime} \cos \phi^{\prime}\right.\right. \\
& \left.+\sin \xi \sin \theta^{\prime}\right)+\cos \theta \sin \phi \cos \theta^{\prime} \sin \phi^{\prime} \\
& \left.-\sin \theta\left(\sin \xi \cos \theta^{\prime} \cos \phi^{\prime}-\cos \xi \sin \theta^{\prime}\right)\right]
\end{aligned}
$$

$$
\begin{aligned}
a_{\phi}\left(\theta^{\prime}, \phi^{\prime}\right)= & \frac{\cos \left(\frac{\pi}{2} \cos \theta^{\prime}\right)}{\sin \theta^{\prime}}\left[\cos \phi \cos \theta^{\prime} \sin \phi^{\prime}\right. \\
& \left.-\sin \phi\left(\cos \xi \cos \theta^{\prime} \cos \phi^{\prime}+\sin \xi \sin \theta^{\prime}\right)\right] .
\end{aligned}
$$

Then we need to transform the above expressions as the functions of $\theta$ and $\phi$. The method adopted here is to treat $\cos \theta^{\prime}, \sin \theta^{\prime}, \cos \phi^{\prime}$ and $\sin \phi^{\prime}$ as independent variables, and directly replaces them with expressions of $\xi, \theta$ and $\phi$. For instance, since $\theta^{\prime}$ is the angle between the unit propagation vector $\hat{\boldsymbol{r}}$ and $\hat{\boldsymbol{z}}^{\prime}$, we have $\cos \theta^{\prime}=\hat{\boldsymbol{r}} \cdot \hat{\boldsymbol{z}}^{\prime}$. After using coordinate transformation, we can express $\hat{\boldsymbol{z}}^{\prime}$ in the GCS, and then obtain $\cos \theta^{\prime}=-\sin \xi \sin \theta \cos \phi+\cos \xi \cos \theta$. Then $\sin \theta^{\prime}$ can be obtained from the relationship of $\cos ^{2} \theta^{\prime}+\sin ^{2} \theta^{\prime}=1$. Similarly, we can express $\cos \phi^{\prime}$ and $\sin \phi^{\prime}$ in the GCS by recognizing the relation of $\cos \phi^{\prime}=\hat{\boldsymbol{r}} \cdot \hat{\boldsymbol{x}}^{\prime} / \sin \theta^{\prime}$. Thus the two components of vectorial element pattern for the rotated dipole can be obtained. For simplicity, we consider the expressions of the two components of vectorial element pattern at XOY plane $(\theta=\pi / 2)$ which is the principal cut of array pattern. They are given as

$$
\begin{gathered}
a_{\theta}(\phi ; \xi)=\frac{\cos \xi \cos \left(\frac{\pi}{2} \sin \xi \cos \phi\right)}{1-\sin ^{2} \xi \cos ^{2} \phi} \\
a_{\phi}(\phi ; \xi)=\frac{\sin \xi \sin \phi \cos \left(\frac{\pi}{2} \sin \xi \cos \phi\right)}{\sin ^{2} \xi \cos ^{2} \phi-1} .
\end{gathered}
$$

Hence, for the linear array with $N$ rotated dipoles shown in Fig. 1, the $\theta$ - and $\phi$-polarized array patterns at XOY plane can be written as

$$
\begin{aligned}
& F_{\theta}(\phi)=\sum_{n=1}^{N} a_{n \theta}\left(\phi ; \xi_{n}\right) e^{j\left(\beta x_{n} \cos \phi+\alpha_{n}\right)} \\
& F_{\phi}(\phi)=\sum_{n=1}^{N} a_{n \phi}\left(\phi ; \xi_{n}\right) e^{j\left(\beta x_{n} \cos \phi+\alpha_{n}\right)}
\end{aligned}
$$

where $j=\sqrt{-1}, \beta=2 \pi / \lambda$ is the wavenumber in free space, $a_{n \theta}\left(\phi ; \xi_{n}\right)$ and $a_{n \phi}\left(\phi ; \xi_{n}\right)$ are $\theta$ - and $\phi$-polarized patterns of the $n$th dipole with a rotation angle of $\xi_{n}$, respectively. $x_{n}$ and $\alpha_{n}$ are the position and excitation phase of the $n$th dipole.

\section{B. Shaped pattern synthesis using dipole rotation and phase optimization}

In order to obtain a desired shaped power pattern for a dipole array with fixed positions, the variables of $\xi_{n}$ and $\alpha_{n}$ for $n=1,2, \ldots, N$ should be optimized with an appropriate fitness function. For the considered dipole array shown in Fig. 1 , the co- and cross-polarized components of the vectorial array pattern in XOY plane are $F_{\theta}(\phi)$ and $F_{\phi}(\phi)$, respectively. Suppose the desired SLL and XPL are $\Gamma_{S L L}$ and $\Gamma_{X P L}$, respectively. The desired co-polarized shaped power pattern is $P_{t}(\phi)$. Then the fitness function can be chosen as follows

$$
\begin{aligned}
f= & \frac{1}{B} \sum_{b=1}^{B}\left\{\left|F_{\theta}\left(\phi_{b}\right)\right|^{2}-P_{t}\left(\phi_{b}\right)\right\}^{2}+\frac{1}{C} \sum_{c=1}^{C} \frac{1}{2}\left(X_{c}+\left|X_{c}\right|\right)^{2} \\
& +\frac{1}{D} \sum_{d=1}^{D} \frac{1}{2}\left(Y_{d}+\left|Y_{d}\right|\right)^{2}
\end{aligned}
$$


where

$$
\left\{\begin{array}{l}
X_{c}=\left|F_{\theta}\left(\phi_{c}\right)\right|^{2}-\Gamma_{S L L}, \quad \phi_{c} \in \text { SLL region } \\
Y_{d}=\left|F_{\phi}\left(\phi_{d}\right)\right|^{2}-\Gamma_{X P L}, \quad \phi_{d} \in \text { XPL region. }
\end{array}\right.
$$

In the above, $\phi_{b}(b=1,2, \ldots, B)$ and $\phi_{c}(c=1,2, \ldots, C)$ are the sampling angles in the shaped beam region and sidelobe region of $F_{\theta}(\phi)$, respectively, and $\phi_{d}(d=1,2, \ldots, D)$ are the sampling angles in the full-space of $\phi \in[0, \pi]$.

Now, the DDE algorithm is adopted to minimize the fitness function by optimizing the variables $\xi_{n}$ and $\alpha_{n}(n=$ $1,2, \ldots, N)$. The DDE inherits all the basic evolutionary operators of CDE but introduces a dynamic evolution mechanism. In every evolution loop of DDE, if a newly generated trial vector is better than the corresponding target individual, it will replace the target individual immediately in the following evolution of the current loop instead of in the next generation. Furthermore, the optimal individual will also be replaced once a newly generated trial vector is better than it. This mechanism makes the DDE perform better at balancing the exploration and exploitation. The reliability and efficiency of the DDE have been shown in different antenna array synthesis applications [20]-[22]. Here, the DDE is applied to optimize element rotation angles and phases for the shaped pattern synthesis with both SLL and XPL control. The proposed synthesis procedure is given in Algorithm 1.

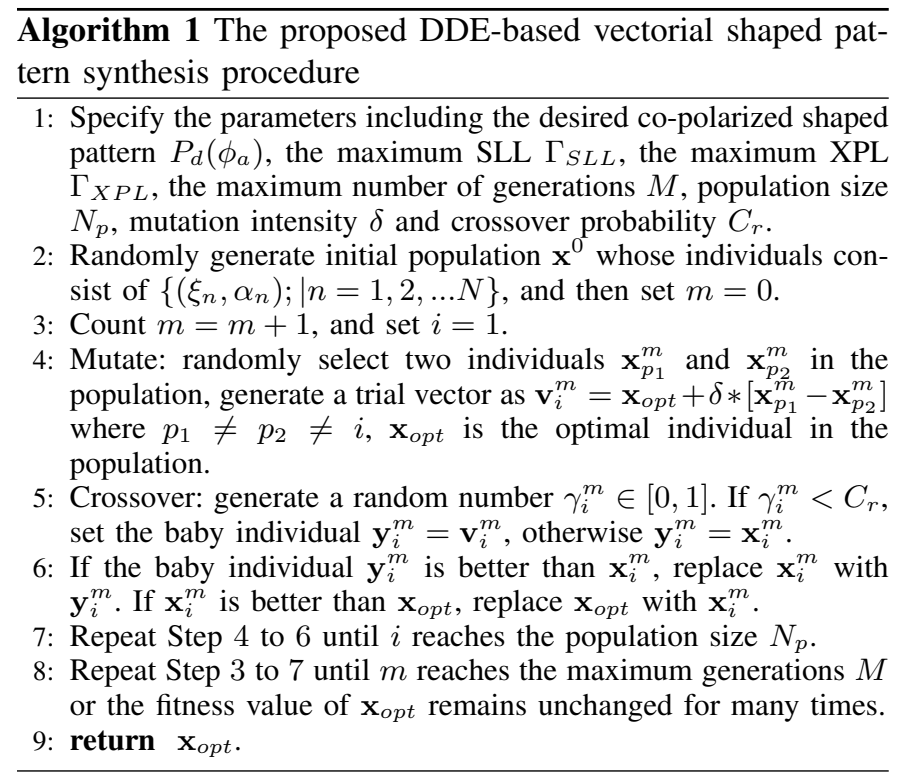

\section{NuMERICAL RESUltS}

In this section, two typical examples for synthesizing a flattop pattern of an equally spaced array and a cosecant-squared pattern of an unequally spaced array are provided to evaluate the performance of the proposed strategy. The comparisons with other methods are also given in the examples.

\section{A. Flat-top pattern synthesis}

As the first example, we consider to synthesize a flattop power pattern which was obtained in [14] by optimizing the phase distribution of a $10 \lambda$-long uniform amplitude line

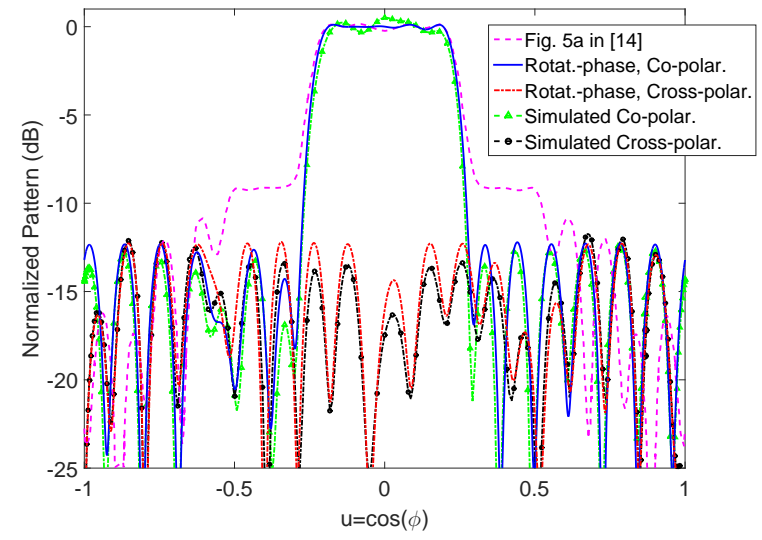

Fig. 2. The synthesized and full-wave simulated co-polarized flat-top patterns and cross-polarized patterns for a 21-element dipole array, and the pattern obtained by phased-only optimization of $10 \lambda$-length linear source in [14].

source. The pattern shown in Fig. 5a of [14] has a flat-top mainlobe with a beamwidth of $28.2^{\circ}$ and a ripple of 0.38 $\mathrm{dB}$, and the obtained sidelobe level (SLL) is $-9.2 \mathrm{~dB}$. Here we try to reproduce this pattern with better performance by simultaneously optimizing the rotation angles and phases of a 21-element $\lambda / 2$-spaced dipole array. In the proposed DDEbased synthesis procedure, we set $\Gamma_{S L L}=\Gamma_{X P L}=-12.5$ $\mathrm{dB}$ for the desired maximum SLL and XPL, $N_{p}=210$ for the population size, $\delta=0.8$ for the mutation intensity, $C_{r}=0.8$ for the cross probability, and $M=3000$ for the maximum number of generations. The synthesized coand cross-polarized patterns as well as the original pattern in [14] are shown in Fig. 2 of this paper. As can be seen, the synthesized co-polarized pattern by the proposed method has a slightly reduced mainlobe ripple (the current ripple is $0.26 \mathrm{~dB}$ ), narrower transition region, and much lower sidelobe distribution. And the obtained maximum SLL and XPL are $-12.32 \mathrm{~dB}$ and $-12.3 \mathrm{~dB}$ which are very close to the specified value. It indicates that the proposed method by using the element rotation and phase optimization has much more degrees of freedom than the phase-only optimization and consequently improves the shaped pattern synthesis performance. The obtained dipole rotation angles and excitation phases are listed in the left column of TABLE I. In addition, the dipole array with the obtained rotation angles and excitation phases is simulated using the full-wave simulation software HFSS. The dipole modeled in the simulation has length of $48 \mathrm{~mm}$ and diameter of $1 \mathrm{~mm}$, working at the frequency of $3 \mathrm{GHz}$. The simulation results are also depicted in Fig. 2. It can be seen that they are generally in accordance with the synthesized patterns except for some fluctuations (about $\pm 0.5 \mathrm{~dB}$ ) in the mainlobe and a litter bit deviation in the sidelobe region. The maximum SLL is almost unchanged and the maximum XPL is increased to $-11.73 \mathrm{~dB}$ from $-12.3 \mathrm{~dB}$ when considering mutual coupling.

\section{B. Cosecant-squared pattern synthesis}

In the second example, the effectiveness of the proposed method is validated for synthesizing an asymmetric shaped 


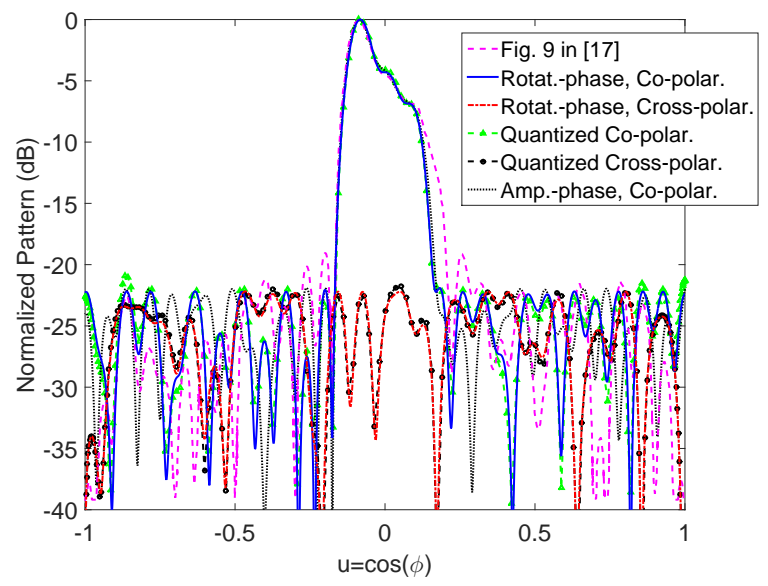

Fig. 3. The synthesized and quantized co-polarized cosecant-squared patterns and cross-polarized patterns by the proposed method, the pattern obtained by the amplitude-phase joint optimization, and the result obtained in [17] for a 29-element unequally spaced linear array.

pattern. A cosecant-squared pattern which was produced in [17] by using 29 nonuniform positions and phases is considered. For comparison, a 29-element dipole array with the same element positions as shown in Fig. 10 of [17] is adopted, and its rotation angles and phases are simultaneously re-optimized by using the proposed method. We choose the cosecantsquared function for the mainlobe region from $\phi=95^{\circ}$ to $\phi=83.5^{\circ}$, and set $\Gamma_{S L L}=\Gamma_{X P L}=-22 \mathrm{~dB}$. The population size is set as $N_{p}=290$, and other parameters are used as in the first example. The synthesized co- and cross-polarized patterns as well as the original pattern in Fig. 9 of [17] are shown in Fig. 3 of this paper. In addition, the result by using amplitude-phase joint optimization for the 29-element unequally spaced array is also given in this example. It is seen that the proposed method has almost the same mainlobe shape but with narrower transition region and much better sidelobe distribution. The amplitude-phase joint optimization has the same co-polarization synthesis performance. However, an amplitude dynamic range ratio of 2.15 is required in this situation. The proposed method does not need to design unequal power dividers. The right column of TABLE I shows the rotation angles and phases obtained by the proposed method. In addition, we also check the effect on the array pattern if both of the rotation angle and excitation phase implemented in practice cannot reach their required accuracies given in TABLE I. For example, suppose the achieved resolution of the mechanical rotation angle is 3 degrees, and the realizable phase quantization interval is 5.625 degrees. The obtained coand cross-polarization array patterns by such quantization are also shown in Fig. 3. As can be seen, the co-polarized shaped mainlobe remains almost the same, but the maximum SLL and XPL increase by $1.07 \mathrm{~dB}$ and $0.33 \mathrm{~dB}$, respectively, due to the quantization. Nevertheless, such performance degradation is usually acceptable in practice.

\section{CONCLUSION}

A novel strategy for synthesizing shaped power patterns by optimizing the element rotation angles and phases of dipole
TABLE I

THE ROTATION ANGLES AND EXCITATION PHASES BY THE PROPOSED METHOD FOR THE FLAT-TOP AND COSECANT-SQUARED PATTERNS

\begin{tabular}{c|cc|cc}
\hline \hline & \multicolumn{2}{|c|}{ Flat-top Pattern } & \multicolumn{2}{c}{ Cosecant-squared Pattern } \\
$\mathrm{n}$ & Rotation $\left(^{\circ}\right)$ & Phase $\left(^{\circ}\right)$ & Rotation $\left(^{\circ}\right)$ & Phase $\left(^{\circ}\right)$ \\
\hline 1 & -167.07 & 172.96 & -95.15 & 214.61 \\
2 & -70.62 & 268.19 & 8.70 & 265.88 \\
3 & 37.41 & 240.71 & 22.61 & 268.05 \\
4 & -11.92 & 224.26 & -58.39 & 260.69 \\
5 & -5.78 & 208.56 & 35.60 & 261.20 \\
6 & 8.17 & 182.04 & -38.70 & 249.25 \\
7 & -3.29 & 169.53 & 57.29 & 253.63 \\
8 & 0.13 & 132.86 & -28.22 & 107.10 \\
9 & -178.71 & 290.60 & 9.10 & 111.77 \\
10 & -2.99 & 100.70 & -14.64 & 97.14 \\
11 & 0.22 & 108.84 & -151.59 & 292.63 \\
12 & -1.74 & 119.77 & 163.33 & 290.81 \\
13 & 0.21 & 163.42 & -177.84 & 290.97 \\
14 & -11.98 & 189.36 & 6.33 & 114.21 \\
15 & 4.93 & 199.08 & -6.80 & 121.01 \\
16 & -14.72 & 217.82 & -7.76 & 111.23 \\
17 & -5.62 & 253.84 & 3.94 & 135.41 \\
18 & -83.77 & 196.25 & -3.96 & 140.56 \\
19 & 141.75 & 147.01 & -9.25 & 129.14 \\
20 & -111.42 & 172.81 & 20.67 & 167.35 \\
21 & 96.78 & 206.76 & -20.85 & 151.39 \\
22 & & & -20.38 & 157.05 \\
23 & & & 35.18 & 179.24 \\
24 & & & -27.92 & 192.17 \\
25 & & & -23.68 & 210.22 \\
26 & & & -175.04 & 202.10 \\
27 & & & -5.56 & 52.58 \\
28 & & & & 264.76 \\
29 & & & \\
\hline \hline
\end{tabular}

array using DDE algorithm has been presented. Compared with the phase-only optimization, the proposed strategy by adding the element rotation technique has much more degrees of freedom to improve the performance of the shaped power pattern synthesis. In addition, this strategy eliminates the need for multiple unequal power dividers and significantly reduces the complexity of the feeding network design. One issue would be the possibly increased XPL which can be controlled by the DDE optimization with an appropriate fitness function. Two examples for synthesizing flat-top and cosecant-squared power patterns are provided, and the comparisons with the phase-only optimization as well as the amplitude-phase joint optimization are also given in the examples.

Finally, it should be noted that although the current formulation is based on linear dipole-rotated array with analytical array pattern expression, the proposed idea capable of synthesizing a shaped pattern by using element rotation and phase optimization can be further extended to general array geometry with arbitrary antenna element structure. In that situation, vectorial element pattern should be obtained using full-wave simulation or measurement. Further research on the extension of the proposed idea is on the way.

\section{REFERENCES}

[1] P. M. Woodward and J. D. Lawson, "The theoretical precision with which an arbitrary radiation pattern may be obtained from a source of a finite size," J. IEE, vol. 95, no. 37, pt. III, pp. 363-370, Sep. 1948.

[2] J.-Y. Li, Y.-X. Qi, and S.-G. Zhou, "Shaped beam synthesis based on superposition principle and Taylor method," IEEE Trans. Antennas Propag., vol. 65, no. 11, pp. 6157-6160, 2017.

[3] W. Stutzman, "Synthesis of shaped-beam radiation patterns using the iterative sampling method," IEEE Trans. Antennas Propag., vol. 19, no. 1, pp. 36-41, Jan. 1971. 
[4] J. L. A. Quijano and G. Vecchi, "Alternating adaptive projections in antenna synthesis," IEEE Trans. Antennas Propag., vol. 58, no. 3, pp. 727-737, Mar. 2010.

[5] K. M. Tsui and S. C. Chan, "Pattern synthesis of narrowband conformal arrays using iterative second-order cone programming," IEEE Trans. Antennas Propag., vol. 58, no. 6, pp. 1959-1970, Jun. 2010.

[6] B. Fuchs, A. Skrivervik, and J. R. Mosig, "Shaped beam synthesis of arrays via sequential convex optimizations," IEEE Antennas Wireless Propag. Lett., vol. 12, pp. 1049-1052, Aug. 2013.

[7] Y. Liu, Z. P. Nie, and Q. H. Liu, "A new method for the synthesis of non-uniform linear arrays with shaped power patterns (Invited Paper)," Progr. Electromagn. Res., PIER, vol. 107, pp. 349-363, 2010.

[8] J. I. Echeveste, M. A. G. de Aza, and J. Zapata, "Shaped beam synthesis of real antenna arrays via finite-element method, floquet modal analysis, and convex programming," IEEE Trans. Antennas Propag., vol. 64, no. 4, pp. 1279-1286, Apr. 2016

[9] F. J. Ares-Pena, J. A. Gonzalez, E. Lopez, and S. R. Rengarajan, "Genetic algorithms in the design and optimization of antenna array patterns," IEEE Trans. Antennas Propag., vol. 47, no. 3, pp. 506-510, Mar. 1999.

[10] P. You, Y. Liu, K. D. Xu, C. Zhu, and Q. H. Liu, "Generalisation of genetic algorithm and fast Fourier transform for synthesising unequally spaced linear array shaped pattern including coupling effects," IET Microw., Antennas Propag., vol. 11, no. 6, pp. 827-832, May 2017.

[11] S. Yang, Y. B. Gan, and P. K. Tan, "A new technique for power-pattern synthesis in time-modulated linear arrays," IEEE Antennas Wireless Propag. Lett., vol. 2, no. 1, pp. 285-287, 2003.

[12] A. A. Akdagli, K. Guney, and D. Karaboga, "Touring ant colony optimization algorithm for shaped-beam pattern synthesis of linear antenna," Electromagnetics, vol. 26, pp. 615-628, 2006.

[13] A. Pirhadi, M. H. Rahmani, and A. Mallahzadeh, "Shaped beam array synthesis using particle swarm optimisation method with mutual coupling compensation and wideband feeding network," IET Microw., Antennas Propag., vol. 8, no. 8, pp. 549-555, Jun. 2014.

[14] A. Trastoy, F. Ares, and E. Moreno, "Phase-only control of antenna sum and shaped patterns through null perturbation," IEEE Antennas Propag. Mag., vol. 43, no. 6, pp. 45-54, Dec. 2001.

[15] L. Marcaccioli, R. V. Gatti, and R. Sorrentino, "Series expansion method for phase-only shaped beam synthesis and adaptive nulling," presented at Proc. URSI Int. Symp. Electromagn. Theory (EMTS), 2004, pp. 676-678.

[16] D. P. Scholnik, "A parameterized pattern-error objective for large-scale phase-only array pattern design," IEEE Trans. Antennas Propag., vol. 64, no. 1, pp. 89-98, Jan. 2016

[17] O. M. Bucci, T. Isernia, and A. F. Morabito, "An effective deterministic procedure for the synthesis of shaped beams by means of uniformamplitude linear sparse arrays," IEEE Trans. Antennas Propag., vol. 61, no. 1, pp. 169-175, Jan. 2013

[18] R. L. Haupt and D. W. Aten, "Low sidelobe arrays via dipole rotation," IEEE Trans. Antennas Propag., vol. 57, no. 5, pp. 1575-1579, May 2009.

[19] M. Li, Y. Liu, S.-L. Chen, P.-Y. Qin, and Y. J. Guo, "Low sidelobe synthesis of dipole arrays by element orientation selection using binary codec genetic algorithm," presented at Proc. EuCAP, 2017, pp. 28382840.

[20] C. Lin, A. Qing, and Q. Feng, "Synthesis of unequally spaced antenna arrays by using differential evolution," IEEE Trans. Antennas Propag., vol. 58, no. 8, pp. 2553-2561, May 2010.

[21] A. Qing, "Dynamic differential evolution strategy and applications in electromagnetic inverse scattering problems," IEEE Trans. on Geoscience and Remote Sens., vol. 44, no. 1, pp. 116-125, Jan. 2006.

[22] P. Rocca, G. Oliveri, and A. Massa, "Differential evolution as applied to electromagnetics," IEEE Antennas Propag. Mag., vol. 53, no. 1, pp. 38-49, Feb. 2011.

[23] K. Woelders and J. Granholm, "Cross-polarization and sidelobe suppression in dual linear polarization antenna arrays," IEEE Trans. Antennas Propag., vol. 45, no. 12, pp. 1727-1740, Dec. 1997

[24] M. Comisso and R. Vescovo, "Fast co-polar and cross-polar 3D pattern synthesis with dynamic range ratio reduction for conformal antenna arrays," IEEE Trans. Antennas Propag., vol. 61, no. 2, pp. 614-626, Oct. 2012 